\title{
La simvastatina disminuye la morbimortalidad cardiovascular inde- pendientemente del nivel de colesterol
}

MRC/BHF Heart Protection Study of cholesterol lowering with simvastatin in 20536 high-risk individuals: a randomised placebocontrolled trial. Heart Protection Study Collaborative Group. Lancet. 2002 Jul 6;360:7-22.

\section{Objetivo}

Evaluar los efectos a largo plazo de la simvastatina en la morbimortalidad vascular y no vascular, en un amplio rango de circunstancias. Diseño

Ensayo clínico controlado aleatorizado (diseño factorial ${ }^{\star}$ ), con un seguimiento promedio de 5 años.

Lugar

Estudio multicéntrico realizado en 69 hospitales del Reino Unido. Pacientes

Hombres y mujeres $(n=20.536)$ entre $40-80$ años con riesgo aumentado de muerte por enfermedad coronaria (EC) a 5 años, basado en antecedentes clínicos:compromiso vascular coronario y no coronario, diabetes, hipertensión y varones mayores a 65 años. Se excluyeron pacientes con: indicación de estatinas por su médico;hepatopatía, compromiso renal;CPK elevada;embarazadas. Intervención

Se aleatorizó a los pacientes para recibir 40mg de SMV $(n=10.269)$ o placebo $(n=10.267)$ una vez por día.Los médicos podían indicar tratamiento hipolipemiante si así lo consideraban.

Medición de resultados principales

El análisis fue por intención de tratar. Se evaluó mortalidad, eventos vasculares fatales y no fatales. Se evaluaron los siguientes resultados combinados: eventos coronarios mayores y eventos vasculares mayores. Resultados principales

El promedio de uso de estatinas fue de $85 \%$ en el grupo SMV y un $17 \%$ en el grupo placebo. La reducción del nivel de LDL en el grupo
SMV fue de $37 \pm 0.8 \mathrm{mg} / \mathrm{dl}$ en relación al placebo.

La mortalidad global se redujo en un $13 \%$ (ver tabla) debido a una disminución significativa del $18 \%$ en la mortalidad por enfermedad coronaria. Se observó una tendencia a la disminución de la mortalidad por otras causas vasculares sin diferencia de muertes por causas no vasculares. La diferencia entre los grupos se observó a partir del segundo año de seguimiento y se mantuvo. No se observaron diferencias significativas en el aumento de enzimas hepáticas, CPK, miopatía, rabdomiolisis, ni en la incidencia de cáncer.

\begin{tabular}{|c|c|c|c|c|c|c|c|}
\hline \multicolumn{2}{|l|}{ EVENTOS } & $\begin{array}{c}\text { Simvastatina } \\
(\mathrm{n}=10269)\end{array}$ & $\begin{array}{c}\begin{array}{c}\text { Placebo } \\
(n=10267)\end{array}\end{array}$ & RR (IC95) & RRA & NNT & $\mathrm{p}$ \\
\hline \multirow[t]{3}{*}{ Mortalidad } & Total & $1328(12.9 \%)$ & $1507(14.7 \%)$ & $0.87(0.81-0.94$ & 1.8 & 56 & 0.0003 \\
\hline & Vascular & $781(7.6 \%)$ & $937(9.1 \%)$ & $0.83(0.75-0.91$ & 1.5 & 67 & 0.0001 \\
\hline & No vascular & $547(5.3 \%)$ & $570(5.6 \%)$ & $0.95(0.85-1.07)$ & & - & NS \\
\hline \multirow{2}{*}{\multicolumn{2}{|c|}{ Evento coronario mayor }} & $898(8.7 \%)$ & $1212(11.8 \%)$ & $0.73(0.67-0.79)$ & 3.1 & 32 & 0.0001 \\
\hline & & $444(4.3 \%)$ & $585(5.7 \%)$ & $0.75(0.66-0.85)$ & 1.4 & 71 & 0.000 \\
\hline $\begin{array}{l}\text { Cualquier ACV } \\
\text { Revascularización }\end{array}$ & ización & $939(9.1 \%)$ & $1205(11.7 \%)$ & $0.76(0.70-0.83)$ & 2.6 & 38 & 0.0001 \\
\hline \multicolumn{2}{|c|}{ Evento vascular mayor } & $2033(19.8 \%)$ & $2585(25.2 \%)$ & $0.76(0.72-0.81)$ & 5.4 & 19 & 0.0001 \\
\hline
\end{tabular}

*Evento coronario mayor:muertes coronarias + IAM no fatal. **Eventos coronarios mayores + cualquier tipo de ACV + cualquier tipo de revascularización

\section{Conclusión}

En población con alto riesgo de eventos cardiovasculares, $40 \mathrm{mg}$ diarios de SMV reducen significativamente la mortalidad cardiovascular y los eventos vasculares mayores. Este beneficio estaría en relación con el riesgo cardiovascular del paciente independientemente del nivel de colesterol basal.

Fuente de financiamiento:UK Medical Research Council, British Herat Foundation, Merck\&Co (fabricantes de simvastatina), Roche Vitamins Ltd.

\section{Comentario}

Estudios observacionales han mostrado que a menor nivel de co lesterol, menor es el riesgo de enfermedad coronaria y que no existe un valor a partir del cual el descenso del colesterol deja de ser beneficioso. Estudios previos ${ }^{1-3}$ con estatinas han mostrado una reducción, del $25 \%$ de los eventos coronarios en pacientes coronarios $\operatorname{con}^{1,2} \mathrm{O} \sin ^{3}$ hipercolesterolemia. Beneficios similares se vieron en pacientes hipercolesterolémicos sin enfermedad coronaria ${ }^{4,5}$.

El Heart Protection Study (HPS) confirma los beneficios previamente observados, brinda nueva información y plantea nuevos interrogantes en cuanto a la terapia con estatinas. Incluyó una amplia población con subgrupos pobremente representados en estudios previos: mujeres, mayores de 75 años, pacientes sin EC, diabéticos sin EC, pacientes con niveles normales de colesterol.Dado que la reducción en los eventos fue similar, y significativa, en todos los subgrupos, el beneficio, y por lo tanto la indicación del tratamiento con estatinas se extiendería a una población mas amplia. Resulta interesante analizar los datos de los pacientes con niveles de colesterol normal. Según las guías del NCEP ATP-III de EE.UU., la población incluida en el HPS debería tener idealmente un LDL $<100 \mathrm{mg} / \mathrm{dl}$. Alrededor de 3500 pacientes tenían un LDL $<100 \mathrm{mg}$ previo a la intervención, y en estos el descenso del nivel de LDL y la reducción de eventos vasculares mayores fue similar al del grupo con niveles mayores de LDL. Los autores plantean que sería mas adecuado guiar la terapia en función del riesgo de eventos vasculares mayores del paciente más que por el nivel de colesterol basal.

Respecto a la seguridad de la SMV, el abandono por reacciones ad- versas fue similar en los dos grupos y se observaron pocos casos de miopatía y de elevación de enzimas hepáticas. A raíz de estos datos algunos autores consideran innecesario el monitoreo con enzimas hepáticas (podría ser útil una determinación basal para identificar elevaciones previas) y solicitar CPK solo a pacientes sintomáticos. En cuanto a la validez externa*, muchos de nuestros pacientes tienen un riesgo vascular similar a la población del HPS. Respecto a la intervención, no es frecuente el uso de SMV a dosis de 40mg/día. Desconocemos cual es el beneficio con dosis menores y es posible que lo observado con SMV sea un efecto de clase para otras estatinas. Cabe mencionar que la cerivastatina fue retirada del mercado por casos frecuentes de miopatía y rabdomiolisis.

Otras intervenciones como la aspirina, los betabloqueantes, los inhibidores de la enzima convertidora de angiotensina han mostrado disminuir, en pacientes de alto riesgo, los eventos vasculares en aproximadamente un tercio cada una. Como se menciona en la editorial $^{6}$, dado que los beneficios de cada intervención parecen ser independientes, combinando estos tratamientos podría obtenerse una reducción del riesgo de eventos vasculares de dos tercios a tres cuartos.Si a esto se suma el control de la tensión arterial en hipertensos y el abandono tabáquico el beneficio podría ser aún mayor.

Conclusión del comentario: En población con alto riesgo cardiovascular, la simvastatina reduce significativamente la mortalidad cardiovascular y los eventos vasculares mayores, en todos los subgrupos incluyendo pacientes con colesterol normal.Sería mas apropiado guiar la terapia en función del riesgo vascular del paciente independientemente del nivel de colesterol basal.

\section{Dr. Santiago Dejo [ Unidad de Medicina Familiar y Preventiva. Hospital Italiano de Buenos Aires ]}

\section{Referencias}

1.Randomised trial of cholesterol lowering in 4444 patients with coronary heart disease:the Scandinavian Simvastatin Survival study (4S). Scandinavian Simvastatin Survival Study Group. Lancet. 1994;344:1383-1389

2.Prevention of cardiovascular events and death with pravastatin in patients with coronary heart disease and a broad range of initial cholesterol levels. The Long-Term Intervention with Pravastatin in Ischemic Disease (LIPID) Study Group.N Engl J Med. 1998;339:1349-1357.

3. The effect of pravastatin on coronary events after myocardial infarction in participants with average cholesterol levels. Sacks FM, Pfeffer MA, Moye LA.N Eng J Med. 1996;335:1001-1009.

4.Prevention of coronary heart disease with pravastatin in men with hyper-cholesterolemia. Shepherd J, Cobbe SM, Ford I, for the West of Scotland Coronary Prevention Study Group.N Eng J Med. 4.Prevention of coronary

5.Primary prevention of acute coronary events with lovastatin in men and women, with average cholesterol levels: results of AFCAPS/TexCAPS. Downs JR, Clearfield M, Weis S, for the AFCAPS/Tex5.Primary prevention of acute coronary events with lovast

6. Two decades of progress in preventing vascular disease. Yusuf S.Lancet 2002 Jul 6;360:2-3. 